\title{
The Role of Hypoxia in Normal Pregnancy and Pregnancy Complications
}

\author{
Nadia Sheibak ${ }^{1, *}$ \\ ${ }^{1}$ Department of Histology, School of Medicine, Zahedan University of Medical Sciences, Zahedan, IR Iran \\ "Corresponding author: Nadia Sheibak, Department of Histology, School of Medicine, Zahedan University of Medical Sciences, Zahedan, IR Iran. Tel: +98-5433295794, Fax: \\ +98-5433295794, E-mail: nadia1989sh@yahoo.com
}

Received 2018 March 10; Accepted 2018 April 23.

\section{Dear Editor,}

Hypoxia is a critical part of normal placentation characterized by $\mathrm{pO}_{2}<20 \mathrm{mmHg}$ in the placenta during the $8^{\text {th }}$ to $10^{\text {th }}$ weeks of pregnancy $(1,2)$.

Invasion of extravillous trophoblast cells (EVTs) to uterine spiral arteries creates the physiologic hypoxia by preventing maternal blood flow into the intervillous space. Decreased oxygen tension in the placenta is essential for the regulation of trophoblastic invasion, proliferation and differentiation, placental angiogenesis, and embryonic organogenesis (1-3).

The mechanism of the regulation and the role of hypoxia in the successful development of the placenta are not clear $(1,3,4)$.

When the maternal blood fills the intervillous space after the $12^{\text {th }}$ week of gestation, placental $\mathrm{pO}_{2}$ increases to 40 $\mathrm{mmHg}$. This increase is required for the maturation of differentiated EVTs (1-3).

Hypoxia-inducible factor 1-alpha (HIF1- $\alpha$ ) is a key regulator for the adaption of the placenta to changes in oxygen pressure and specially EVTs differentiation. Normal developments of the placenta and fetus are depended on the HIF1- $\alpha$ modulation and cellular defense against oxidative stress $(2,4)$. The expression of HIf- 1 and TGFb3 molecules is high in early pregnancy before the $10^{\text {th }}$ week, while after that, it falls when placental $\mathrm{pO}_{2}$ levels are believed to increase. In fact, the oxygen-regulated early events of trophoblast differentiation are in part mediated by TGFb3 through HIF-1 transcription factors (5).

Any change in the occurrence or duration of physiologic hypoxia could result in pregnancy complications. Some possible mechanisms are the loss of differentiating stimulator (physiologic hypoxia) or cellular damage in the presence of the excess reactive oxygen species. It could result in the abnormal depth and extent of invasion by EVTs that are regulated by oxygen tension normally $(1,2,6)$.

Placental oxygen level during pregnancy is associated with increased and decreased cytotrophoblast proliferation and differentiation rates to regulate normal placentation. It is shown that several pregnancy complications vary from miscarriage to preeclampsia caused by dysregulation of oxygen pressure in the placenta $(1,2)$.

In addition, the structural changes of the placenta in some pregnancy complications might be related to hypoxia. We found that in some maternal disorders such as lupus erythematous and smoking that were able to induce non-physiological hypoxia, the development and histological structure of the placenta and differentiation of EVTs could be affected (7-9).

The effect of hypoxia on the differentiation and invasion of EVTs was reported in the several in-vitro studies. However, the molecular mechanism of invasion regulation by the hypoxia is poorly understood that needs more investigation in future research. In addition, until now, there are limited numbers of in-vivo investigations on this topic $(1,2)$.

However, in-vitro findings of the molecular mechanisms of EVTs invasion may come close to the in-vivo condition; however, placental adaptations that occur physiologically should be considered. On the other hand, it is demonstrated that in-vivo placental oxygen level and duration of different stages of gestation may not be provided in the in-vitro model (2). Therefore, there is a necessity for more focus on this mechanism using other methods and techniques that are able to give us more data about the invivo process of trophoblastic differentiation (9).

As some molecular mechanisms of pregnancy complications were investigated in the previous issues of "Gene, Cell, and Tissue" (10), I suggest that immunohistochemistry, real-time PCR, and other advanced techniques $(7,9)$ can be employed for the identification of the association between hypoxia, placental development, and pregnancy complications in the physiological in-vivo conditions. It could be the subject of future studies in this field. 


\section{References}

1. Zhao H, Jiang Y, Cao Q, Hou Y, Wang C. Role of integrin switch and transforming growth factor Beta 3 in hypoxia-induced invasion inhibition of human extravillous trophoblast cells. Biol Reprod. 2012;87(2):47.1-7.

2. Tuuli MG, Longtine MS, Nelson DM. Review: Oxygen and trophoblast biology-a source of controversy. Placenta.2011;32 Suppl 2:S109-18. doi: 10.1016/j.placenta.2010.12.013. [PubMed: 21216006]. [PubMed Central: PMC3682830].

3. Wakeland AK, Soncin F, Moretto-Zita M, Chang CW, Horii M, Pizzo D, et al. Hypoxia Directs Human Extravillous Trophoblast Differentiation in a Hypoxia-Inducible Factor-Dependent Manner. Am J Pathol. 2017;187(4):767-80. doi: 10.1016/j.ajpath.2016.11.018. [PubMed: 28167044]. [PubMed Central: PMC5397702].

4. Horii M, Li Y, Wakeland AK, Pizzo DP, Nelson KK, Sabatini K, et al. Human pluripotent stem cells as a model of trophoblast differentiation in both normal development and disease. Proc Natl Acad Sci U S A. 2016;113(27):E3882-91. doi: 10.1073/pnas.1604747113. [PubMed: 27325764]. [PubMed Central: PMC4941448].

5. Caniggia I, Mostachfi H, Winter J, Gassmann M, Lye SJ, Kuliszewski M, et al. Hypoxia-inducible factor-1 mediates the biological effects of oxy- gen on human trophoblast differentiation through TGFbeta(3). J Clin Invest. 2000;105(5):577-87. doi: 10.1172/JCI8316. [PubMed: 10712429] [PubMed Central: PMC289179].

6. Wallace AE, Fraser R, Cartwright JE. Extravillous trophoblast and decidual natural killer cells: a remodelling partnership. Hum Reprod Update. 2012;18(4):458-71. doi: 10.1093/humupd/dms015. [PubMed: 22523109]. [PubMed Central: PMC3373213].

7. Heidari Z, Mahmoudzadeh-Sagheb H, Sheibak N. Quantitative changes of extravillous trophoblast cells in heavy smoker mothers compared with healthy controls. Reprod Fertil Dev. 2018;30(2):409-14 doi: 10.1071/RD17041. [PubMed: 28723341].

8. Heidari Z, Mahmoudzadeh-Sagheb H, Sheibak N. Placenta structural changes in heavy smoking mothers: a stereological aspect. Curr Med Res Opin. 2018:1-5. doi:10.1080/03007995.2018.1444590. [PubMed: 29469634].

9. Heidari Z, Mahmoudzadeh-Sagheb H, Sheibak N, Nourzaei N. Quantitative changes of extravillous trophoblast cells in placentas of systemic lupus erythematosus patients.JObstet Gynaecol.2017;37(6):74651. doi: 10.1080/01443615.2017.1306695. [PubMed: 28399698].

10. Fazli M, Ghorbian S. Association Study of Non-Coding RNA miR-499 and miR196a2 Gene Polymorphisms with the Risk of Idiopathic Recurrent Pregnancy Loss. Gene, Cell and Tissue. 2018;In Press(In Press). 